\title{
Chair Inserts for Preschoolers
}

\author{
1 \\ Eva Horn \\ Caren E. Millen \\ Christie Lee Cavanaugh \\ Shelley Komisar
}

Preschool children with physical impairments have special furniture and equipment needs (Connors, Williamson, \& Siepp, 1978). Adaptive seating equipment for these children is crucial to their physical and educational development (Bergen, 1974). Proper adaptive seating equipment should inhibit abnormal reflexes and muscle tone, while simultaneously facilitating more normal body movement (Bobath, 1969).

\section{Background}

The primary objective in developing an adaptive seating system is to maximize the student's motor development and independence in functional activities (Bergen, 1974). The adaptive seat should be readily available, reasonably priced, and individualized to the student's physical needs. It must be flexible, adapting to changes in the child's size, motor abilities, and functional needs as well as in his or her social environment.

These requirements led the teaching staff of our demonstration class for infants and toddlers with severe and multiple handicapping conditions to investigate the feasibility of building adaptive chairs using double-walled cardboard. Their effort resulted in inexpensive chair inserts that can either be placed in commercially made cube chairs or attached to other types of preschool chairs. The physical positioning and support needs of individual children were determined under the supervision of a staff physical therapist, and specific design features for meeting these needs were provided by a consulting occupational therapist. The chairs were constructed by the teaching staff inexpensively $(\$ 5$ to $\$ 10$ each) and with minimal construction time (approximately 2 hours each). The procedures the teachers followed are described in this article. It is important to note, however, that although teachers can follow the instructions for construction, a physical or occupational therapist should be consulted during the motor assessment of the child to determine the child's positioning needs (i.e., the type and amount of support needed for an optimal sitting position) and to guarantee accurate chair measurements.

\section{Design Steps}

Adaptive equipment is, by definition, customized to meet the physical needs of individual children. In order to customize the equipment, a blueprint . for construction must first be developed: This involves three steps: (a) assessing current sitting status; (b) designing equipment that provides a balance of support; and (c) obtaining the measurements for the insert. The design should prevent the child from falling into nontherapeutic positions and force the child to attempt to maintain a correct sitting posture.

\section{Step 1: Assess Current Sitting Status}

The first step is to assess the child's current sitting position by placing the child in a sitting position with as little support as necessary. A description of the child's hip position, trunk alignment, head position, and general body symmetry should be developed in consultation with a physical or occupational therapist. This information is used to determine the child's optimal sitting position and to decide what support is needed to achieve the ideal sitting position. Considerations include approximating normal hip position, trunk elongation and symmetry, total body symmetry, and active maintenance of an upright sitting position (Hobson \& Trefler, 1981).

\section{Step 2: Provide a Balance of Support}

In consultation with a physical or occupational therapist, the next step is to determine what type of structural support is needed to maintain the optimal sitting position. Emphasis should be placed on providing as little support as possible, to allow for the child's active participation in maintaining the correct position but not allow the child to fall into nontherapeutic positions.

\section{Step 3: Obtain Measurements for the Insert}

Insert measurements are derived by measuring the child as he or she is held in the prescribed optimal sitting position. The types of child measurements needed and the procedure for determining the chair measurements from them are shown in Figure 1. In the remainder of the article the upper case 


\section{Figuŕe 1}

ADAPTIVE INSERT MEASUREMENTS

Name:

Date:

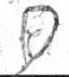

Measured by:

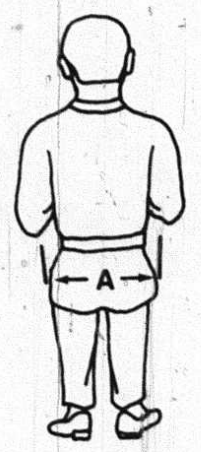

Child Measurements

$A=$ (hip to hip)

$\mathrm{B}=$ — (top of shoulder to tailbone)

$\mathrm{C}=$ — (tailbone to back of knee)

$\mathrm{D}=$ _ (back of knee to heel)

$E=\ldots$ (foot length)

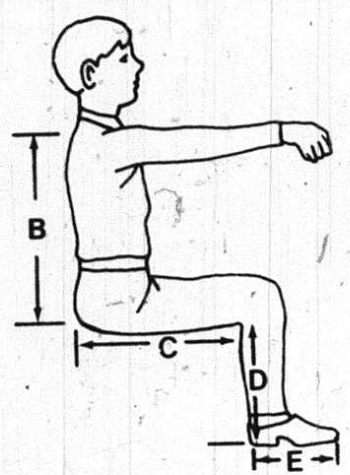

Chair Measurements

a. Seat Width $=-(A+1$ inch $)$

b. Back Height $=$ (Based on

trunk support needed)

c. Seat Depth $=$ - (C - 1/2 inch $)$

d. Leg Rest Length $=$ - (D)

e. Foot Pedal Length $=\ldots(E+2)$
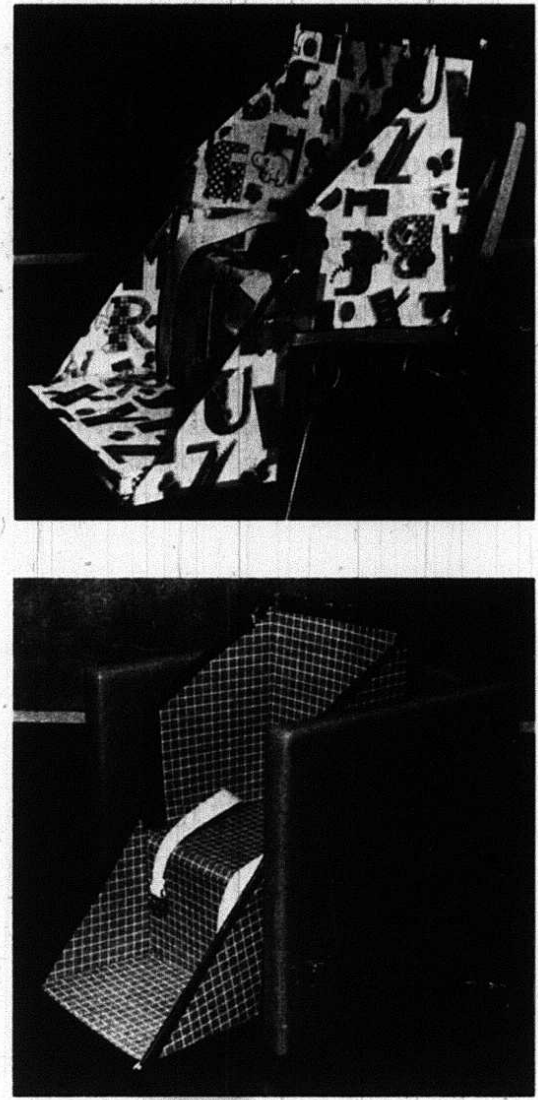

\section{Figure 3}

\section{ASSEMBLY}

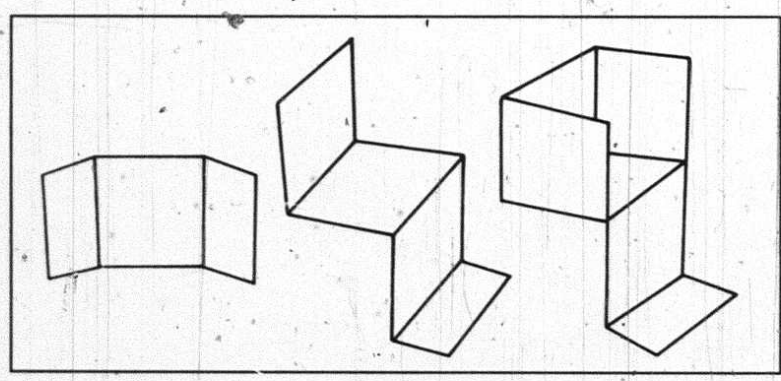

(a) Take main chair frame and combine with back and side supports.
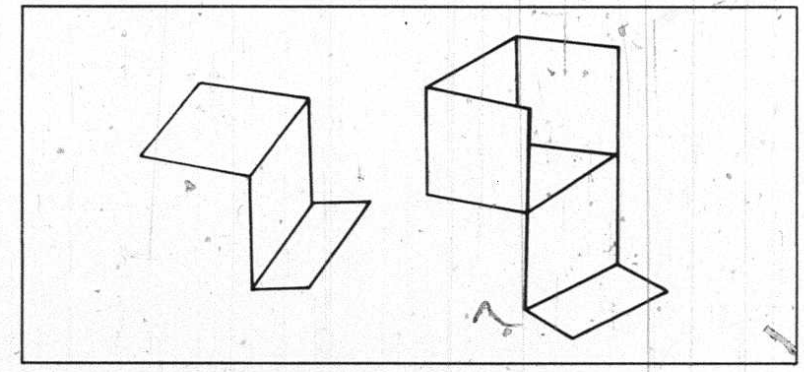

(b) Add seat and leg rest to assembled chair frame and back and side supports.

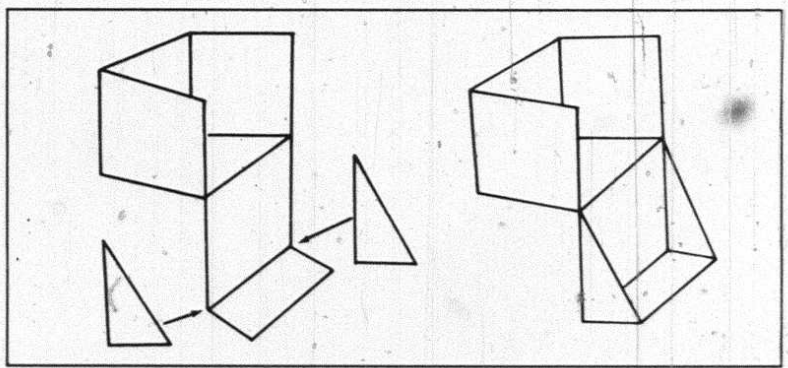

(d) Add sides for footrest.

(c) Add bottom and side support.

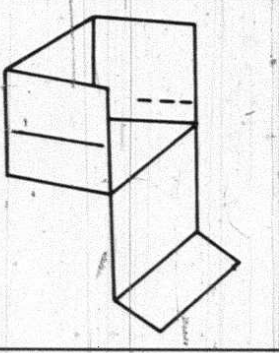


Figure 2

MEASURING, CUTTING, AND BENDING THE CARDBOARD

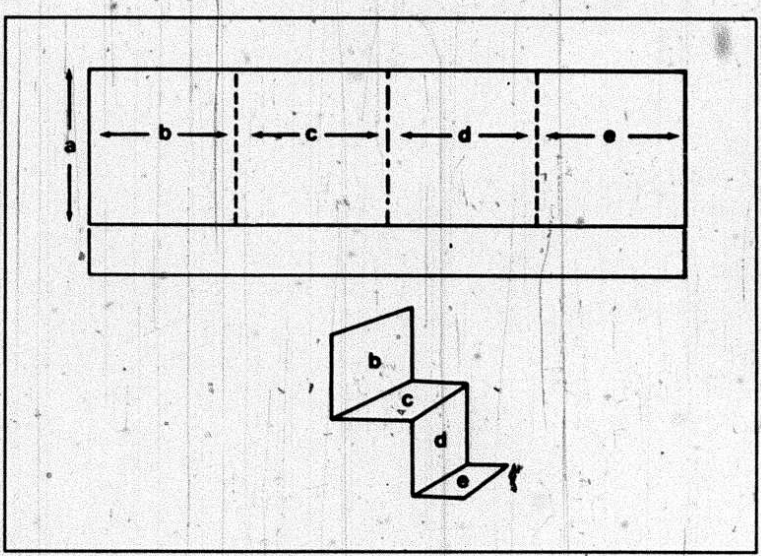

(a) Main chair frame.

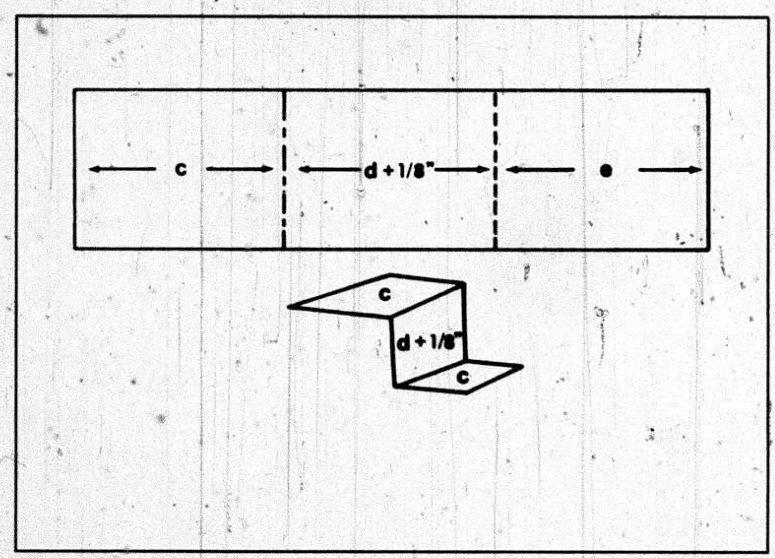

(c) Seat and leg rest.

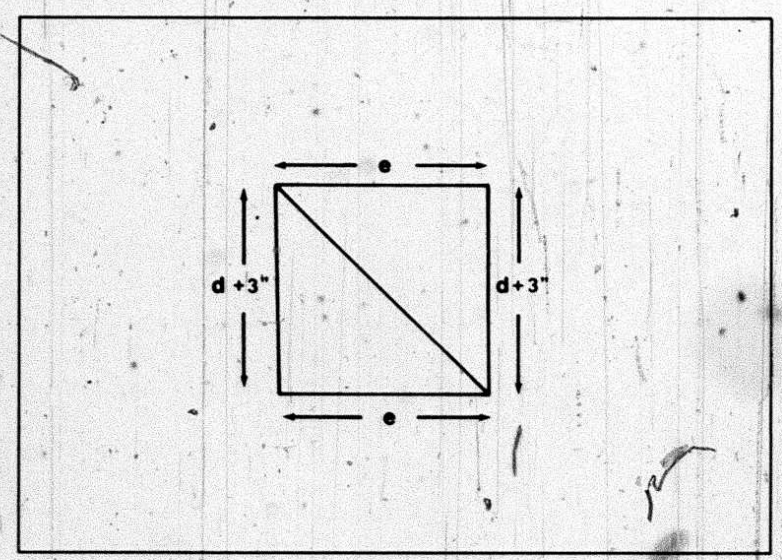

(e) Sides for footrest.

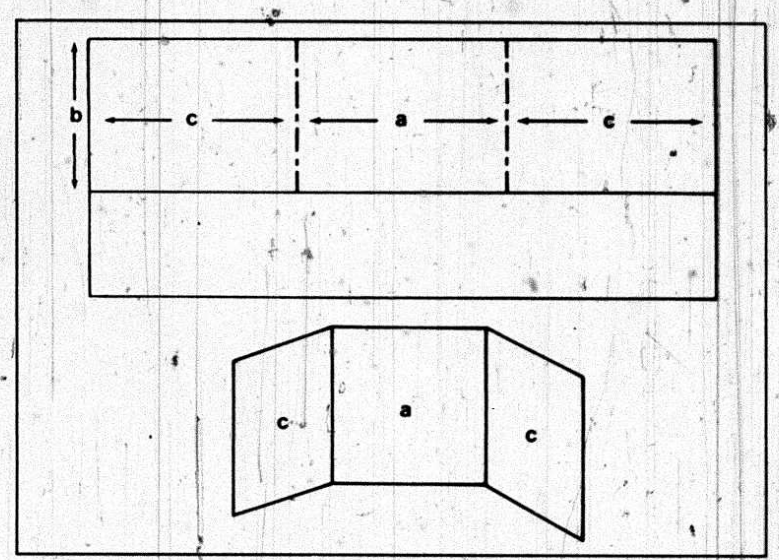

(b) Back and side supports.

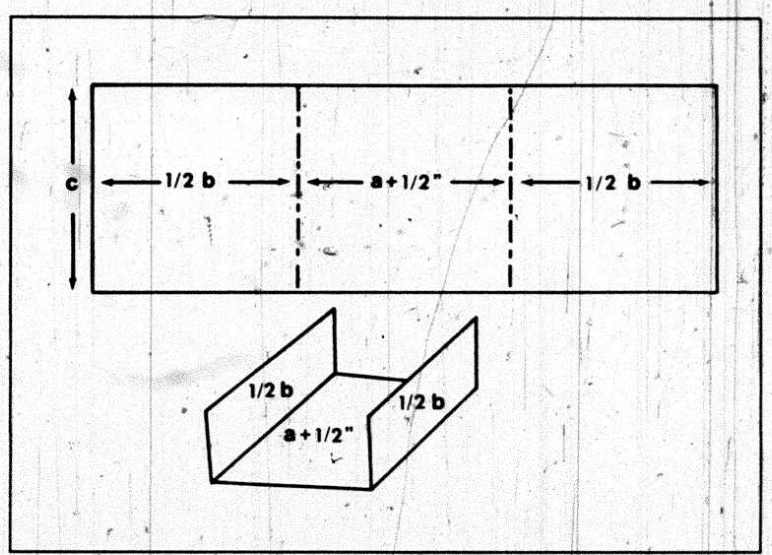

(d) Bottom and side support.

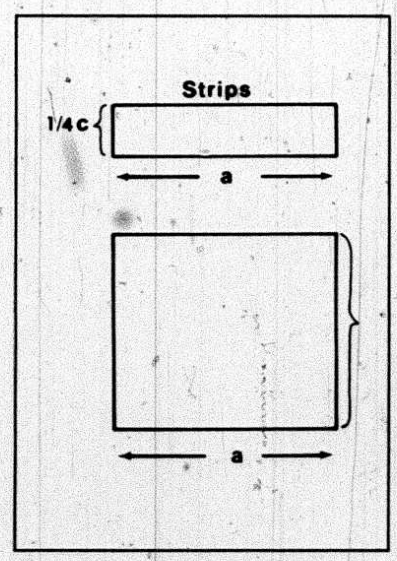

Cut number of strips needed for height of roll.

This length measurement is determined by the number: of strips used; must be wide enough to cover stack of strips plus front and back edges formed by stack.

Figure Key

Solid line indicates clean cut through cardboard.

Dotted line indicates light cut through one layer for bend on underside of cardboard.

..............-. Dash/dot line indicates light cut through one layer for bend on topside of cardboard. 
letters in the figures refer to actual child measurements, and lower case letters refer to child measurements that have been converted to chair measurements as shown in Figure 1 .

\section{Construction Steps}

The materials needed for constructing these chair inserts are:
- list of child/insert measurements
- heavy duty tape
- carpenter's wood glue
- contact paper
- double-walled cardboard
- velcro straps
- webbing for the strap

The tools needed for constructing these chair inserts are:

- straight-edged ruler or angle for transferring measurements onto the cardboard

- exacto knife

- pencil or pen to mark measurements on the cardboard

- scissors

Gather all materials and locate yourself in a large, open area with ample work space. An ideal construction material is the corrugated double-walled cardboard found in large appliance storage boxes, which is sturdy enough to support the child.

\section{Measuring, Cutting, and Bending the Cardboard}

Each insert is constructed by assembling six parts: the main chair frame; back and side support; seát and leg rest support; bottom and side support; sides for the footrest; and seat roll. Diagrams for measuring, cutting and bending each of these pieces are presented in Figure 2.

If a roll in the front of the seat under the child's knees is necessary to push the hips back and maintain hip flexion, consult Figure 2(f). Enough strips of cardboard should be cut to obtain the desired height of the roll. They should be glued on top of each other and then to the front part of the seat. Mold the larger piece from the seat roll, bending it repeatedly along the cardboard's natural grooves to form a curve. Then place the curve over the stack of strips on the front edge of the chair, insert seat to form a half roll.

\section{Preliminary Assembly}

The pieces are now ready to be assembled. Figure 3 depicts the order and procedures for putting pieces together. Masking tape should be used to hold the pieces together before they are permanently affixed with glue.

Front edges of the insert sides may be cut off to allow for free arm movements, while still giving support and stability to the shoulder area.

\section{Finishing Surfaces}

Inserts may be covered with contact paper to enhance their attractiveness. After the chair is temporarily taped together, mark the exposed surfaces with an $X$ or other symbol. Once the exposed areas have been marked, take the chair apart and apply the contact paper to the marked surfaces only. The unexposed surfaces must not be covered because they will be glued together and the glue does not bond as well to contact paper as to cardboard.

\section{Final Assembly}

Refer to Figure 3 and apply glue to one of the two surfaces to be attached. As each piece is attached, use tape to secure the edges. This serves as a clamp while the glue dries. The sides for the footrest need to be securely attached with heavy duty tape because there are no adequate gluing surfaces (See Figure $3[d]$ ). This is also true when attaching the roll covering to the seat bottom.

\section{Durability}

The durability of a cardboard chair insert will depend on the individual child's activity level and growth. The 12 inserts we have constructed have lasted from 6 months to 1 year. They have had to be reconstructed due to the children's growth and changes in support needs. Small to medium spills wipe up nicely because of the contact paper covering. However, occasions may occur in which a large amount of spillage will saturate the chair, requiring partial rebuilding or reinforcement of various components. Because of the multiple layers of cardboard and the wrapping effect in construction, the chairs have proved quite durable even for a child with high muscle tone and strength.

\section{Summary :}

Adaptive seating equipment for preschool children with physical impairments is crucial to their physical and educational development. Obtaining proper seating, however, can be expensive and time consuming. This article has provided detailed procedures for constructing cardboard chair inserts to meet the needs of children with minimal to severe physical limitations. These inserts offer a number of advantages including reduced expense, reduced construction time, and increased flexibility which allows a customized fit.

\section{References}

Bergen, A. (1974). Selected equipment for pediatric rehabilitation. Valhahla NY: Blythedale Children's Hospital.

Bcbath, B. (1969). The treatment of neuromuscular disorders by improving patterns of coordination. Physiotherápy, 55, 18-22.

Connors, F., Williamson, G., \& Siepp, J. (1978). Program guttle for infants and toddlers with neuromotor and other developmental disabilities. New York: Teachers College Press:

Hobson, D., \& Trefler, E. (1981). MPI System: Clinical application manual. Memphis: University of Tennessee, Rehabilitation Engineering Center.

Eva Horn (CEC Chapter \#242) is a doctoral fellow in the Department of Special Education; Caren E. Millen (CEC Chapter \#242) is Replication Coordinator in the Infant Toddler Learning Project; Christie Lee Cavanaugh is Parent Trainer in the Infant Toddlet Learning Project; and Shelley Komisar is Curriculum Coordinator, all at the Kennedy Center Experimental School, Peabody College of Vanderbilt University, Nashville, Tennessee.

Support for the development activities discussed in this article was provided through a model demonstration grant from the Handicapped Children's Early Education Program, Specjal Education Programs, U.S. Department of Education (\#G008302259)

Copyright 1987 CEC. 